\title{
Constraining Atom Probe Tomography Reconstructions of Crystalline Oxides
}

\author{
M. J. Cohen ${ }^{1}$, L. M. Gordon ${ }^{1}$, S. Suram ${ }^{2}$, K. Kaluskar ${ }^{2}$, K. Rajan ${ }^{2}$, and J. W. Valley ${ }^{3}$, D. Joester ${ }^{1}$ \\ ${ }^{1}$ Department of Materials Science and Engineering, Northwestern University, Evanston, IL 60208. \\ ${ }^{2}$ Department of Materials Science and Engineering and Institute for Combinatorial Discovery, Iowa \\ State University, Ames, IA 50011 \\ ${ }^{3}$ Department of Geoscience, University of Wisconsin, Madison, WI 53706 USA
}

Atom-probe tomography (APT) is uniquely capable of directly probing the location and chemical identity of the atoms within a sample of material with sub-nanometer spatial resolution and parts-permillion chemical sensitivity. APT consists of a field-ion microscope coupled to a time-of-flight mass spectrometer, providing chemical and three-dimensional positional information of ions successively field evaporated from a sharp sample. While early application of APT was limited to electrically conductive metals and heavily doped semiconductors recent advancements in ultraviolet laser pulsing has enabled the analysis of a range of dielectric materials [1].

While APT provides a detailed picture of the distribution of elements in three-dimensions there are a number of parameters that control how the impact position and sequence of ions are translated into the final 3D reconstruction. In pure metals and elemental semiconductors field evaporation processes result in multiple low-density regions observed crystallographic poles. These regions are also often accompanied by lattice-plane resolution in the dataset. The presence of multiple poles and lattice plane resolution provides key information to constrain the reconstruction algorithm [2].

In complex multi-component materials, fewer crystallographic poles and lattice planes are observed in reconstructions, with only a few examples in the literature to date [3]. We report here on the combination of sample orientation, X-ray and electron diffraction and advanced data analysis to calibrate reconstruction parameters from single- and nano-crystalline oxide materials, including geological and biogenic samples [4]. Geological magnetite single crystals were oriented with faces parallel to the (100), (110) and (111) planes using Laue backscatter X-ray diffraction and electron backscatter diffraction. Oriented crystals were cut with a slow speed diamond saw and polished with successively finer grits of silicon carbide abrasive discs and diamond suspension. Standard focused ion beam lift out and tip sharpening protocols were then used to prepare tips from the polished surfaces.

APT of the oriented samples revealed poles only along the $<111>$ directions. No $<100>$ or $<110>$ poles were observed. When only a single $<111>$ pole is visible only lattice spacing can be used to constrain reconstructions. However, in the case of a $<110>$ oriented sample, two $<111>$ poles are observed close to the edges of the field of view. In this scenario, the known crystallographic angle between the poles and lattice spacing are combined to further contrain the reconstruction. We will also discuss the use of nanostructural features within single crystal samples with a known orientation relative to the sample crystallography to further constrain reconstructions.

[1] T.F. Kelly, D.J. Larson, Ann. Rev. Mat. Res. 2012, 42, 1-31

[2] B.P. Gault et al. J. Appl. Phys. 2009, 105, 034913

[3] B.P. Gault et al. Materials Today. 2012, 15, (9) 378-386

[4] L.M. Gordon, D. Joester, Nature 2011, 469, 194-197 
Figure 1. Left. 2D ion density maps of APT reconstructions for the (100), (110) and (111) oriented magnetite crystals, only <111> poles are visible as low-density regions (black arrows, numbered). Three-fold symmetry is observed in the (111) oriented sample. Center. Corresponding Laue backscatter $\mathrm{X}$-ray diffraction of magnetite single crystals and Right. Simulated diffraction patterns.
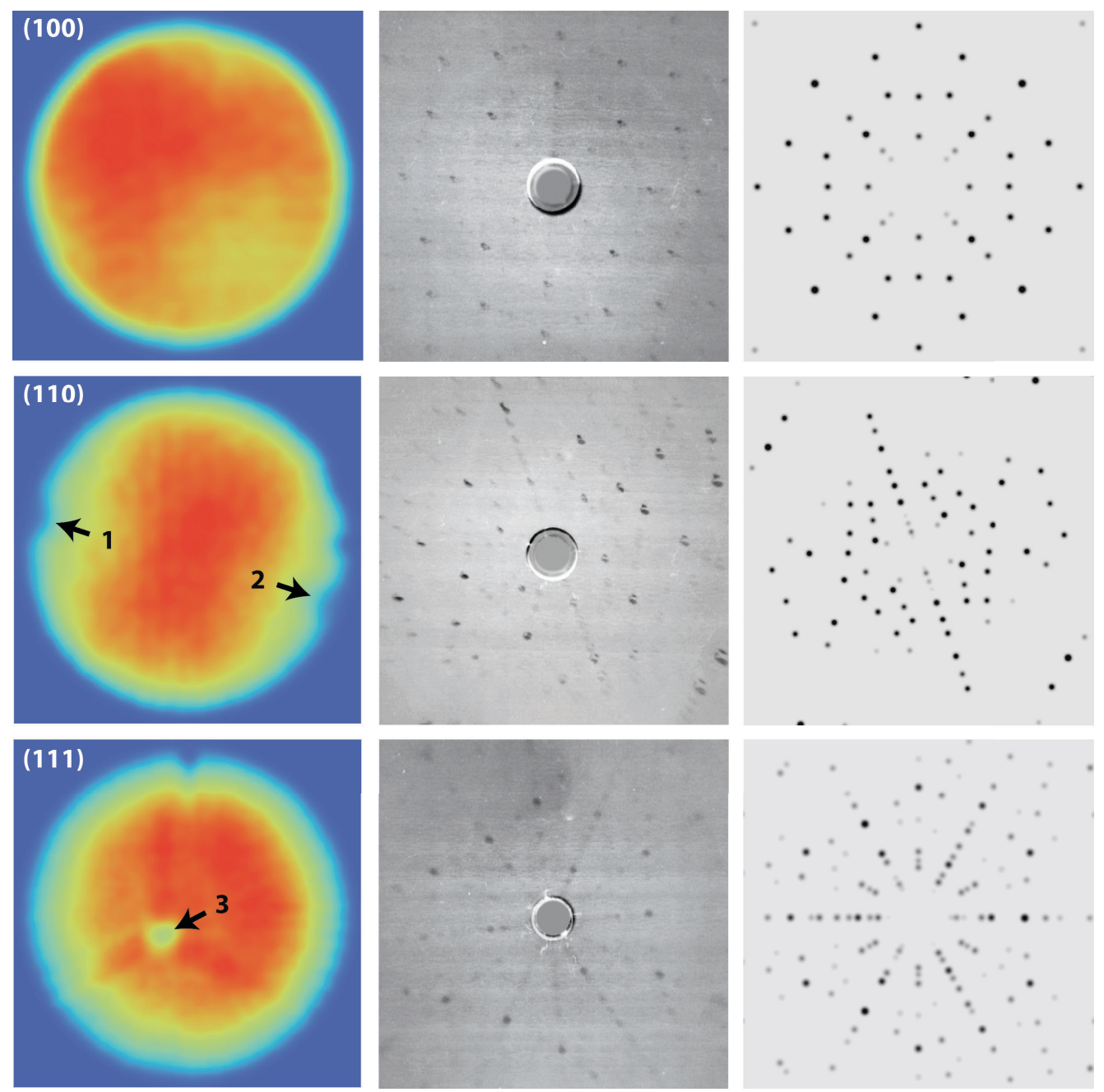

Figure 2. APT 3D reconstructions corresponding to numbered $<111>$ poles in Figure 1 with (111) lattice planes visible in the regions surrounding the poles.
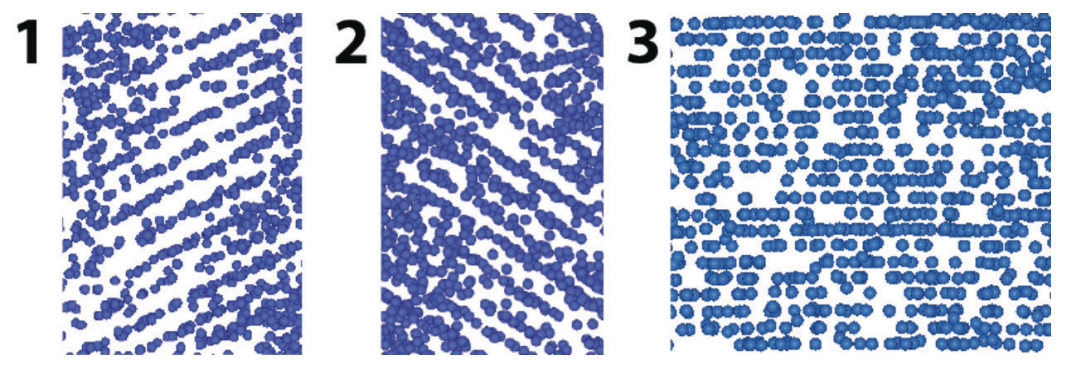